\title{
La existencia de necesidades de información y comportamiento informativo en los entrenadores en deportes de combate
}

\author{
Salvador Enrique VÁzQUEZ-MocTEZUMA \\ Universidad Nacional Autónoma de México \\ quique.vazquez@hotmail.com
}

Recibido: 31/05/2015

Aceptado: 21/09/2015

\section{RESUMEN}

Este trabajo tiene como propósito esencial, realizar un acercamiento para detectar e identificar las necesidades de información y el comportamiento informativo de entrenadores en deportes de combate. Para ello se aplicó un cuestionario a instructores de aikido, boxeo, esgrima, judo, karate, kendo, lima lama, lucha y taekwondo seleccionados mediante un muestreo no probabilístico por causalidad. En general encontramos que los principales temas de interés entre los instructores son: los programas de entrenamiento, nutrición y dietas de entrenamiento. Por otra parte, los entrenadores son más propensos a utilizar su experiencia, internet y cursos para obtener información. En contraste se nota que la biblioteca y los libros son poco usados.

Palabras clave: Necesidades de información, comportamiento informativo, búsqueda de información, entrenador, deportes de combate, conocimiento.

\section{Information needs and information-seeking behavior of combat sport coaches}

\begin{abstract}
This paper has as main purpose, make an approach to detect and identify information needs and information-seeking behavior of coaches in combat sports. Was applied a questionnaire to teachers of aikido, boxing, fencing, judo, karate, kendo, lima lama, wrestling and taekwondo selected by a non-probability sampling causality. In general, we find that the main topics of interest among instructors are training programs, nutrition and training diets. Moreover, coaches are more likely to use their experience, internet and courses for information. In contrast, we note that the library and the books are rarely used.

Keywords: Information needs, information behavior, search for information, coach; combat sports, knowledge.

\section{Referencia normalizada}

Vázquez-Moctezuma, S.E (2015). “La existencia de necesidades de información”. Documentación de las Ciencias de la Información, Vol. 38: páginas. 103-123
\end{abstract}




\section{INTRODUCCIÓN}

Las bibliotecas son lugares de enlace entre la información y el usuario, por esto tienen la misión principal de satisfacer las necesidades informativas. Aunque, para poder lograr esa función se requiere saber quiénes son y no los usuarios, qué información les interesa y cuál es su comportamiento para obtener aquella. Así los profesionales de la información realizan estudios de usuarios, con el fin de tomar mejores decisiones en el desarrollo de colecciones además de crear, modificar o eliminar algún servicio de información. Es evidente que en varias bibliotecas se carece de algún tipo de estudio de usuarios, pero la existencia de alguno de estos que se acerque a las características de la biblioteca puede ser punto de partida para aquellas bibliotecas que no tienen definido a los usuarios a los que atiende.

Hay una amplia gama de unidades de información que se enfocan en diferentes grupos sociales como los deportistas, además los sistemas de clasificación LC en la clase G y Dewey en la clase 700 consideran los temas de deportes. En la ciudad de México existen menos de una docena de bibliotecas especializadas en deportes (Iglesias Anaya, 2011), que consideran como sus principales usuarios a los entrenadores, deportistas, y profesionistas de las ciencias aplicadas al deporte. Actualmente estas bibliotecas presenta la problemática constante de la conexión con los entrenadores. Por lo cual, el no tener clara las necesidades y comportamiento de los instructores en deportes se asociaría a la ausencia de estos en la biblioteca.

En cuanto al rol del entrenador deportivo en la sociedad tiene funciones bien definidas, Vargas (1998) lo considera como el especialista que dirige todo el proceso de dirección pedagógica del entrenamiento deportivo, para ello planifica, organiza, controla, analiza y evalúa todas las actividades concernientes a este proceso, de modo que, enseña una disciplina deportiva, determina el programa y las actividades para el aprendizaje, coordina las sesiones de entrenamiento, verifica el perfeccionamiento de ejecución de las técnicas aplicadas, propone estrategias de acción en competencias, corrige errores y deficiencias cometidas en la práctica del atleta, aconseja en relación a la dieta requerida, establece las cargas de trabajo, entre otras actividades.

Por su parte, García (2006) menciona que ser entrenador incluye un conjunto de tareas tanto implícitas como explicitas. Éstas, comprenden su interrelación con el cuerpo técnico, comisión directiva, atletas, periodistas, padres de familia y fanáticos del deporte. Como se observa el instructor deportivo juega un papel destacado en los grupos sociales por todas las actividades que desempeña y el impacto que tiene ante otros individuos.

En las ciencias de la información a nivel internacional son limitadas las investigaciones publicadas sobre las necesidades y comportamiento informativo en entrenadores de deporte y las pocas que existen se enfocan en entrenadores que están en alguna etapa de formación (Clarke, 1995; Guðmundsson, 2011). Sin embargo un estudio reciente de Vazquez y Calva (2013), encontraron que muchos deportistas amateurs acuden con el entrenador para buscar información en vez de usar la biblioteca. Mien- 
tras que desde la perspectiva de las ciencias del deporte y medicina del deporte, hay algunas publicaciones que tratan ambiguamente el tema del comportamiento informativo de los deportistas, aunque no han podido establecer sus necesidades de información y el comportamiento en la búsqueda de información, ya que no son su objeto de estudio. Considerando que el factor de desarrollo en el deporte y las primeras iniciativas del estudio científico se produjeron en los años cincuenta aunque, fue hasta la década de 1960 cuando las ciencias del deporte adquirieron una identidad propia debido a la creciente importancia del deporte como fenómeno social, cultural, político y económico (Remans, 2000).

Lo planteado anteriormente permite visualizar que se tienen bibliotecas especializadas en deportes también que su principal usuario son los entrenadores, a pesar de que éste individuo no use en su totalidad la unidad de información, ocupa un lugar destacado en la sociedad debido a sus funciones que afectan al grupo social al que pertenece. Con motivo de la carencia de publicaciones sobre las necesidades de información y comportamiento informativo de los entrenadores, éste trabajo será pionero en esta área de investigación, pues se enfocará en entrenadores que se desempeñan como instructores deportivos y que no se encuentran en formación. También brindará acercarse a un sector social hermético y poco tratado por la documentación, al menos en Latinoamérica, es decir a los entrenadores dedicados a la instrucción de los deportes de combate (aikido, boxeo, esgrima, judo, karate, kendo, lima lama, lucha y taekwondo), lo cual ofrecerá un bosquejo para aquellas unidades de información que desconocen al entrenador como usuario de la información.

Así que hemos generado los siguientes cuestionamientos: ¿Cuáles son las necesidades de información de los entrenadores en deportes de combate? y ¿Qué fuentes y recursos de información utilizan para cubrir sus necesidades de información? Con base en estas interrogantes la investigación exploratoria y cuantitativa se delineo como propósito detectar e identificar las necesidades de información de los entrenadores de atletas en deportes de combate, así como los recursos y fuentes de mayor uso en su búsqueda de información, para ello se aplicó un cuestionario a 28 instructores en deportes de combate seleccionados mediante un muestreo no probabilístico por causalidad de dos instituciones de educación superior de la Ciudad de México y el estudio de campo se efectuó en los gimnasios del campus central de la Universidad Nacional Autónoma de México y el Instituto Politécnico Nacional.

Naturalmente el estudio de los instructores deportivos podría brindar información relevante para el desarrollo de colecciones también para construir, modificar o eliminar servicios y sistemas de información con base en las necesidades y comportamiento informativo expresada por los propios entrenadores en ésta investigación. 


\section{LAS NECESIDADES DE INFORMACIÓN DEL ENTRENADOR DEPORTIVO}

El concepto sobre necesidades de información de acuerdo con Sanz Casado (1994) es la sensación de la carencia de algo que se origina una vez satisfecho los requerimientos básicos de alimento, sed, alojamiento, sexo, etc., de modo que las necesidades de información se generan una vez cubierto las necesidades básicas o fisiológicas. En contraste Hernández Salazar (2001) considera que la necesidad informativa es la capacidad del ser humano por reconocer la existencia de una incertidumbre; e información como aquel estímulo capaz de reducirla.

Para Wilson una necesidad es una experiencia subjetiva que ocurre únicamente en la mente de la persona que tiene una necesidad y, consecuentemente, no es directamente accesible para ser observada (Wilson, 2000). Por su parte Krikelas (1983) menciona que una necesidad de información es la función de la incertidumbre extrínseca producida por una discrepancia percibida entre los niveles corrientes de certeza acerca de objetos importantes del entorno y un estado de criterio que busca alcanzar esos niveles de certeza. Entonces las necesidades de información son subjetivas y generadas cuando el sujeto detecta su limitada información sobre algo que al quererla aumentar esa información manifiesta un comportamiento; es decir, una transformación en sus actividades para reducir su carencia y proveerse de información.

Las investigaciones publicadas que se ha llevado a nivel internacional referente a las necesidades de información de la comunidad de entrenadores en las ciencias de la información son escasas y específicamente, nulas en lo relativo a instructores de deportes de combate. Sin embargo, Clarke (1995) advierte que los principales temas de interés para los instructores es la información sobre motivación de atletas y programas de entrenamiento.

Por otra parte, existen algunos trabajos en las ciencias del deporte que tratan superficialmente el tema de las necesidades informativas entre la comunidad deportiva. La literatura advierte que los entrenadores necesitan información y conocimiento sobre temas de planificación y enseñanza de las técnicas deportivas (Ermeling, 2012; Jones, 2006). Psicología del deporte, es decir, dirección, orientación y control de la actividad de los deportistas, así como la motivación (García, 2006; Sagar y Jowett, 2012), pues el entrenador de atletas tiene la función de enseñar una disciplina deportiva.

Las lesiones en el deporte ocurren comúnmente durante el ejercicio, las cuales son resultado de accidentes, otras debido a limitadas prácticas de entrenamiento, equipo inapropiado, falta de condición física, calentamiento insuficiente o excesivo (Hart, Trivedi, y Hutchinson, 2012; Salerno,2009). Entonces, el conocimiento de la prevención y rehabilitación de lesiones implica información que debe poseer o buscar en un momento dado el entrenador. 
El tema de nutrición, hidratación y consumo de suplementos alimenticios en el ambiente deportivo ocupa un lugar destacado porque, el hombre al realizar una actividad física necesita energía la cual es obtenida a través del consumo de alimentos, cuando el atleta tiene una alimentación insuficiente así mismo un arduo entrenamiento o viceversa es seguro que el desempeño sea deficiente , también al realizar una actividad física el cuerpo empieza a sudar y por lo tanto a perder líquidos de ahí que se necesite la rehidratación para continuar con un buen desempeño (Christine, 2012; Pettersson et al, 2012; Pumpa et al., 2012; Torres-McGehee, 2012). En los deportes de contacto con frecuencia se compite por categorías de peso, sin embargo, para su cuidado es indispensable una alimentación balanceada, es de suponerse que el entrenador necesite información de nutrición y suplementos alimenticios ya que en muchos casos es el encargado de vigilar el peso del atleta.

Por otro lado, cuando se realizan competencias o torneos, es común que se practiquen distintos exámenes médicos a los deportistas; entre los de mayor controversia están los de antidopaje, cuya finalidad es detectar el uso de sustancias ilícitas o drogas que incrementan la condición física (Hanstad y Loland, 2009; Hanson, 2009; Thomas et al, 2011). Otro aspecto que no puede faltar es lo concerniente a la legislación deportiva (Wong, 2010), pues en el deporte se siguen reglas de juego o competición, asimismo sanciones, derechos y obligaciones de asociaciones deportivas, códigos de conducta, reglamentos, entre otros.

Sin dudad, el entrenador debe poseer aptitudes de enseñanza de un pedagogo, los conocimientos de un buen doctor, los dotes de mando de un ejecutivo empresarial y está en condiciones de impartir sabios consejos de un psicólogo experimentado (Martens et al, 1995). Existen algunas necesidades de información comunes y otras tan específicas entre los entrenadores, generando que se vuelva una comunidad de estudio compleja y completa, de modo que pueden presentar un comportamiento en la búsqueda de información que los obligará a requerir de distintas fuentes y recursos de información especializada en su deporte.

\section{EL COMPORTAMIENTO EN LA BÚSQUEDA DE INFORMACIÓN DEL ENTRENADOR DEPORTIVO}

Con respecto al comportamiento informativo Wilson lo define como la totalidad del comportamiento humano en relación a los recursos y canales de información, abarcando la búsqueda de información, activa y pasiva, y el uso de información. Incluye, tanto la comunicación cara a cara con otros, como la recepción pasiva de información (Wilson, 2000). Sin embargo, Krikelas (1983) señala que el comportamiento es cualquier actividad de un individuo dirigida a identificar un mensaje que satisfaga una necesidad percibida como tal. Aunque Kuhlthau (1991), concibe al proceso de búsqueda de información como la actividad constructiva que un sujeto realiza para encontrar sentido a la información, con el fin de aumentar su conocimien- 
to sobre un tema o problema específico. Así concluimos que el comportamiento informativo es el reflejo de la existencia de alguna necesidad informativa además es la acción donde el individuo intenta desaparecer o reducir su ignorancia a través de la información almacenada en su memoria y otros recursos informativos que le ayuden a entender su necesidad.

Sin duda, haber practicado el deporte es la forma más sencilla de que los entrenadores adquieren información y conocimiento sobre técnicas, reglas, estrategias y un sinfín de temas. Aunque, a veces haber practicado el deporte no concede todo el conocimiento que hace falta, ni tampoco no haberlo practicado significa que no pueda adquirirse éste conocimiento. Entonces el entrenador en un momento dado tendrá alguna necesidad informativa, es decir, detectará su limitada información sobre algo y al quererla aumentar manifestará un comportamiento de búsqueda en cualquier fuente, documento y soporte para satisfacer su necesidad de información.

Desde la perspectiva de las ciencias de la información, al menos en Latinoamérica, se desconoce el comportamiento en la búsqueda de información de distintas comunidades no académicas, entre ésta la de los entrenadores deportivos. Un acercamiento próximo al comportamiento informativo en la comunidad de atletas en deportes de contacto es el estudio de Vazquez y Calva (2013) revelo que los principales recursos y fuentes de búsqueda de información entre los boxeadores amateurs son: el entrenador, otros atletas, internet y casi en última instancia la biblioteca. También Clarke (1995) identifico que la biblioteca no es el principal recurso para obtener información entre los instructores en natación y atletismo en Australia. Por su parte, Guðmundsson (2011) encontró que los entrenadores en natación utilizan diferentes medios para buscar información, como libros, revistas, conferencias, revistas y la World Wide Web.

Aunque un estudio de Fowler (1976) en el cual examino a 1200 profesores de educación física pudo detectar que los libros de texto y conferencias eran los principales recursos informativos. Un trabajo de Guy (1979) inspecciono a 79 investigadores en deportes, educación física y recreación donde observo que los recursos principalmente usados eran las publicaciones periódicas, libros, reportes de investigación, consulta con colegas y otras instituciones, pero los recursos que menos usan son fotografías, videos y películas, así mismo concluye que probablemente el comportamiento informativo de los investigadores podría ser diferente al de los entrenadores.

Escasos estudios en ciencias del deporte señalan que los entrenadores en general, no específicamente instructores de deportes de combate, buscan y obtienen información así como conocimiento en distintos medios. Los trabajos de Cushion (2001), Cushion et al, (2003), Gilbert y Trudel (2001) y Gould et al, (1990) consideran que la experiencia y la observación de otros entrenadores son los principales recursos de conocimiento para los entrenadores. Aunque Callary et al, (2012) ubica los aspectos anteriores agrega también, que el entrenador adquiere conocimientos de la experiencia con atletas, cursos, seminarios o conferencias además de la familia. No obstante, Wright y sus colegas (2007) indican que libros, videos e internet son medios para 
adquirir conocimiento. El deporte se encuentra rodeada de especialistas como médicos, nutriólogos, psicólogos, masoterapeutas, fisioterapeutas, entre otros, los cuales están allí para practicar más inteligentemente las actividades deportivas mismos que pueden brindar información al entrenador (Lévesque,1991). Por su parte, Wilson (2008) y Höferlin et al, (2010) mencionan que el video ha sido utilizado por el entrenador para observar el desempeño de los atletas y con esto hacer ajustes en sus técnicas y habilidades.

Como se observa en la literatura en las ciencias de la información y ciencias del deporte se ha identificado las fuentes y recursos informativos que utilizan los entrenadores que se encuentran en alguna etapa de formación pero se desconoce el comportamiento informativo del entrenador que forma atletas.

\section{METODOLOGÍA}

Para llevar a cabo esta investigación exploratoria y cuantitativa se aplicó un cuestionario como instrumento en dos instituciones de educación superior de la Ciudad de México y el estudio de campo se efectuó en los gimnasios del campus central de la Universidad Nacional Autónoma de México así como en el Instituto Politécnico Nacional con el propósito de recolectar los datos necesarios. Lamentablemente se carece de un directorio nacional sobre el número de gimnasios donde se practique deportes de combate aunado a esto la comunidad de entrenadores es hermética.

Se eligieron esas dos instituciones educativas por que se encuentran en un contexto académico lo que permitiría facilitar la investigación asimismo imparten la mayoría de los deportes de combate y en un caso, la universidad, cuenta con biblioteca especializada en deportes mientras que en el instituto existe geográficamente próxima una biblioteca especializada en deporte.

Para llevar a cabo esta investigación en un primer momento se buscó en la página Web de la Dirección General de Actividades Deportivas y Recreación de la Universidad Nacional Autónoma de México UNAM ${ }^{1}$ asimismo, en la página de deportes del Instituto Politécnico Nacional IPN ${ }^{2}$ para determinar el número de deportes de combate y de entrenadores, además de la ubicación física de los gimnasios. La población corresponde así a 31 individuos y 9 deportes al ser una población pequeña se decidió por no elaborar una muestra.

El instrumento en forma de cuestionario se aplicó del 10 al 28 de noviembre de 2014 antes y después de las sesiones de entrenamiento. Cabe mencionar que no se

\footnotetext{
${ }^{1}$ Página oficial de los deportes impartidos en la UNAM en: http://www.deportes.unam.mx/disciplinas/

${ }^{2}$ Página oficial de los deportes impartidos en el IPN en:

http://www.deportes.ipn.mx/deportes/Paginas/Disciplinas.aspx
} 
ejecutó el cuestionario con los entrenadores de judo y taekwondo del Instituto Politécnico Nacional por que se encontraban participando con sus atletas en una competencia en el Estado de Veracruz, México. Por lo cual 28 cuestionarios fueron llenados debidamente por instructores en aikido, boxeo, esgrima, judo, karate, kendo, lima lama, lucha y taekwondo de la UNAM y el IPN.

\section{RESULTADOS}

Los 28 entrenadores encuestados pertenecen a nueve deportes de combate: aikido (1), boxeo (5), esgrima (2), judo (1), karate (6), kendo (1), lima lama (2), lucha (5) y taekwondo (5). Como se observa en la tabla 1, la mayoría de los instructores son hombres $(89 \%, \mathrm{n}=25)$. Con respecto a la edad, el (43\%) menciono tener más de 50 años. En cuanto al nivel académico, se encontró que la mayoría de los entrenadores tiene estudios de licenciatura $(61 \%, n=17)$, cabe mencionar que ningún instructor se formó en ciencias del deporte, de modo que se formaron empíricamente. Por otra parte, en términos de experiencia cerca de un tercio (32\%) de los entrenadores cuenta con más de 30 años de experiencia. A su vez, el (29\%) respondió que enseña a la semana más de 28 horas.

Tabla 1. Perfil de los entrenadores en deportes de combate ( $\mathrm{n}=28)$

\begin{tabular}{lcc}
\hline & $N$ & $\%$ \\
\hline Genero & & \\
Masculino & 25 & 89 \\
Femenino & 3 & 11
\end{tabular}

Edad

25 a 30 años

11

31 a 35 años

$\begin{array}{cc}3 & 11 \\ 4 & 14 \\ 4 & 14 \\ 2 & 7 \\ 3 & 11 \\ 12 & 43\end{array}$

36 a 40 años

14

41 a 45 años

7

46 a 50 años

11

Mayor de 50 años

12

Educación

Preparatoria

$\begin{array}{ll}2 & 7\end{array}$

Carrera Técnica

$6 \quad 21$

Licenciatura

17

61

Maestría

3

11

Experiencia

Menos de 5 años

$3 \quad 11$

Entre 5 a 10 años

$4 \quad 14$ 
Entre 11 a 15 años

Entre 16 a 20 años

Entre 21 a 25 años

Entre 26 a 30 años

Más de 30 años

$\begin{array}{cc}3 & 11 \\ 5 & 18 \\ 3 & 11 \\ 1 & 1 \\ 9 & 32\end{array}$

Tiempo semanal empleado en la enseñanza deportiva

4-8 horas

9-13 horas

14-18 horas

19-23 horas

24-28 horas

Más de 28 horas
11

18

11

32

\subsection{Tipos de necesidades de información de los entrenadores}

Los instructores deportivos se pueden cuestionar un sin número de cosas de lo que perciben en su ambiente, de ahí que sus necesidades informativas sean de diferentes tipos. Sin embargo, hay algunos temas que son de interés común entre la comunidad de entrenadores en deportes de combate, en el gráfica 1 se exhibe una lista en orden de prioridad de las necesidades de información, gran parte de los entrenadores $(n=26)$ mostraron gran interés por la materia de programas de entrenamiento, nutrición y dietas. Aunque, los tópicos de motivación en atletas $(n=25)$ y técnicas de combate $(n=24)$ también ocupan un lugar destacado. En contraste, menos del $(25 \%)$ de los encuestados mostro desinterés sobre asuntos del conocimiento de rivales u oponentes, productos y equipos de competencia, bebidas rehidratantes, records deportivos, sedes de competencia, rutas y medios de transporte. 
Gráfica 1. Necesidades informativas de los entrenadores en deportes de combate

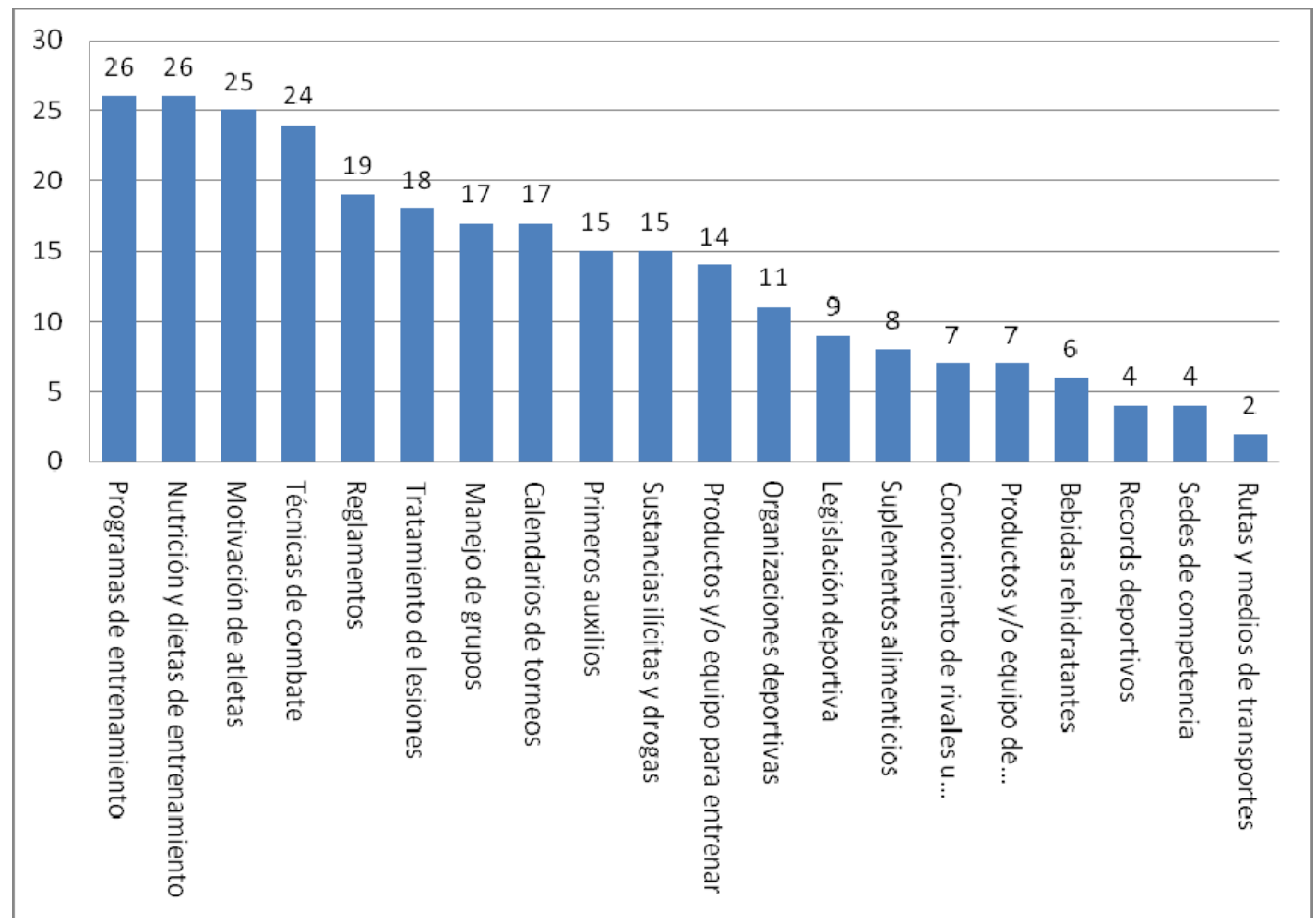

Las necesidades de información mencionadas anteriormente no son las únicas, ya que $(46 \%, n=13)$ de los entrenadores advirtió tener otras inquietudes específicas. En la tabla 2 se añaden otros tipos de información pertinente para la comunidad en deportes de combate. 
Tabla 2. Otro tipo de información de interés para los entrenadores

\begin{tabular}{|c|c|}
\hline - $\quad$ Programas de becas y estímulos & - $\quad$ Eventos de intercambio de \\
\hline económicos para atletas y entrenadores & entrenadores de diversos países \\
\hline Infraestructura y arquitectura depor- & $\begin{array}{l}\text { - Sociología del deporte (en } \\
\text { general) }\end{array}$ \\
\hline Psicología del deporte (en general) & Historia de cultura marcial \\
\hline $\begin{array}{l}\text { - } \\
\text { estadística para control en competencias }\end{array}$ & $\begin{array}{l}\bullet \quad \text { Tramites de importación de } \\
\text { armas }\end{array}$ \\
\hline Aspectos filosóficos del karate & $\begin{array}{l}\text { - Metodología del entrenamien- } \\
\text { to deportivo }\end{array}$ \\
\hline $\begin{array}{l}\text { - Test psicológicos para atletas (en } \\
\text { general) }\end{array}$ & Técnicas de meditación \\
\hline
\end{tabular}

\subsection{Comportamiento en la búsqueda de información de los entrenadores}

De acuerdo a los datos reportados por los entrenadores en deportes de combate se muestra en la tabla 3 la frecuencia de uso de los distintos recursos para la búsqueda de información bajo los parámetros: frecuentemente, poco y nunca usado. 
Tabla 3. Total de coincidencias de uso de los recursos de información a los que acuden los entrenadores $(n=28)$

\begin{tabular}{lccc}
\hline Recursos de información & $\begin{array}{c}\text { Frecuentemente } \\
\text { usado }\end{array}$ & $\begin{array}{c}\text { Pocas veces } \\
\text { usado }\end{array}$ & $\begin{array}{c}\text { Nunca } \\
\text { usado }\end{array}$ \\
\hline Otros entrenadores & 15 & 8 & 5 \\
$\begin{array}{l}\text { Conocimiento propio (expe- } \\
\text { riencia) }\end{array}$ & 24 & 2 & 2 \\
$\begin{array}{l}\text { Atletas } \\
\text { Biblioteca, centro de docu- } \\
\text { mentación }\end{array}$ & 8 & 9 & 11 \\
$\begin{array}{l}\text { Colección personal de libros } \\
\text { y revistas }\end{array}$ & 21 & 15 & 5 \\
Jueces y árbitros & 7 & 5 & 2 \\
$\begin{array}{l}\text { Médicos, nutriólogos, etc. } \\
\text { Masoterapeuta y fisiotera- }\end{array}$ & 12 & 6 & 15 \\
$\begin{array}{l}\text { peuta } \\
\text { Psicólogo }\end{array}$ & 12 & 14 & 2 \\
$\begin{array}{l}\text { Internet (google, wikipe- } \\
\text { dia,blogs, etc.) }\end{array}$ & 14 & 9 & 7 \\
Familiares y amigos & 23 & 10 & 4 \\
Cursos, conferencias, talleres & 2 & 3 & 2 \\
\hline
\end{tabular}

La mayor parte de instructores indica que recurre a su experiencia personal $(n=24)$ como primer recurso informativo, enseguida acude a internet $(\mathrm{n}=23)$ así como a cursos, conferencias y talleres $(\mathrm{n}=22)$. En oposición la biblioteca por muchos entrenadores $(70 \%, n=20)$ no es usada o poco explotada para obtener información que les exige su ambiente deportivo, sin embargo la mayoría $(n=21)$ consulta su colección personal. Por otro lado, se recurre pocas veces a los psicólogos, médicos, árbitros y atletas para buscar información (véase la gráfica 2). 
Gráfica 2. Grados de uso de recursos de información

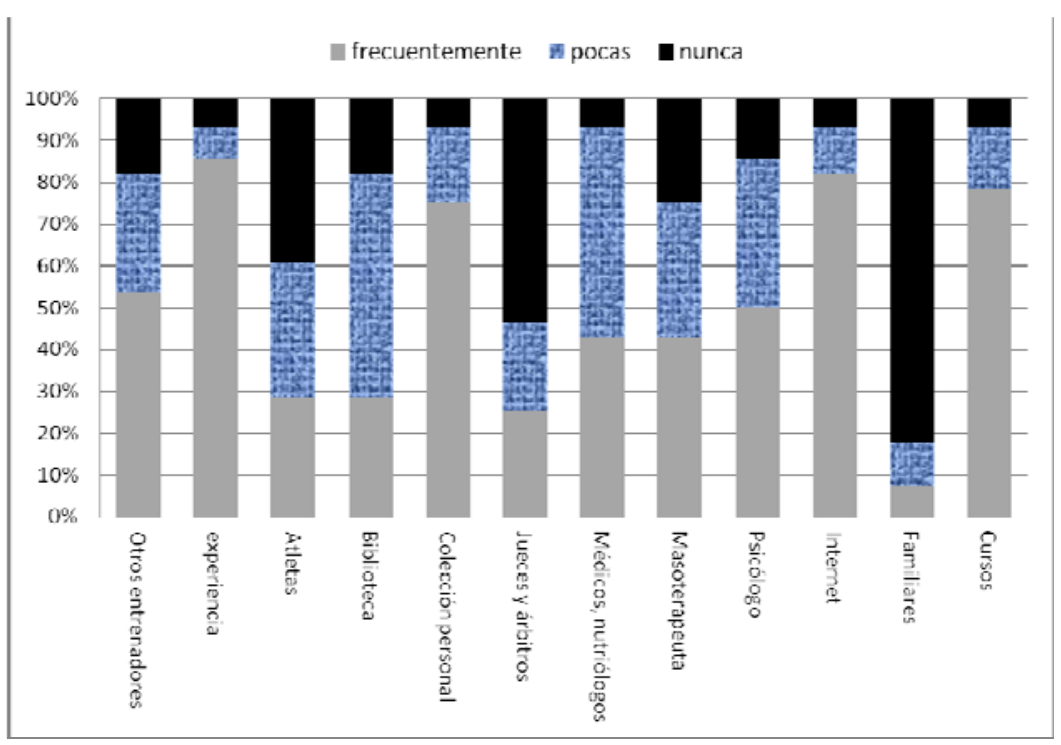

Por otra parte, los entrenadores no solo utilizan recursos informativos, sino tambien se apoyan en diversas fuentes (véase la gráfica 3). Lo que encontramos fue que cerca de dos tercios del total de los entrenadores $(n=19)$ opino que con frecuencia utiliza los reglamentos deportivos, tambien los videos ocuparon un lugar destacado ya que alrededor de $(60 \%, \mathrm{n}=17)$ instructores confirmaron este hecho.

En seguida, los libros tambien son bastante usados, sin embargo los entrenadores mencionaron que muchos de estos son de su colección personal $(n=16)$.En contraste, mas del 60\% de los entrenadores en deportes de comabte manifesto que la radio y el periódico son las fuentes que nunca usan para obtener información. Existe una gran catidad de fotografias defiguras en deportes de combate, pero es limitada la información que contienen, por lo cual, más de la mitad de los instructores $(n=16)$ mencionaron que poco las consultan. 
Gráfica 3. Frecuencia de uso de fuentes de información entre los entrenadores

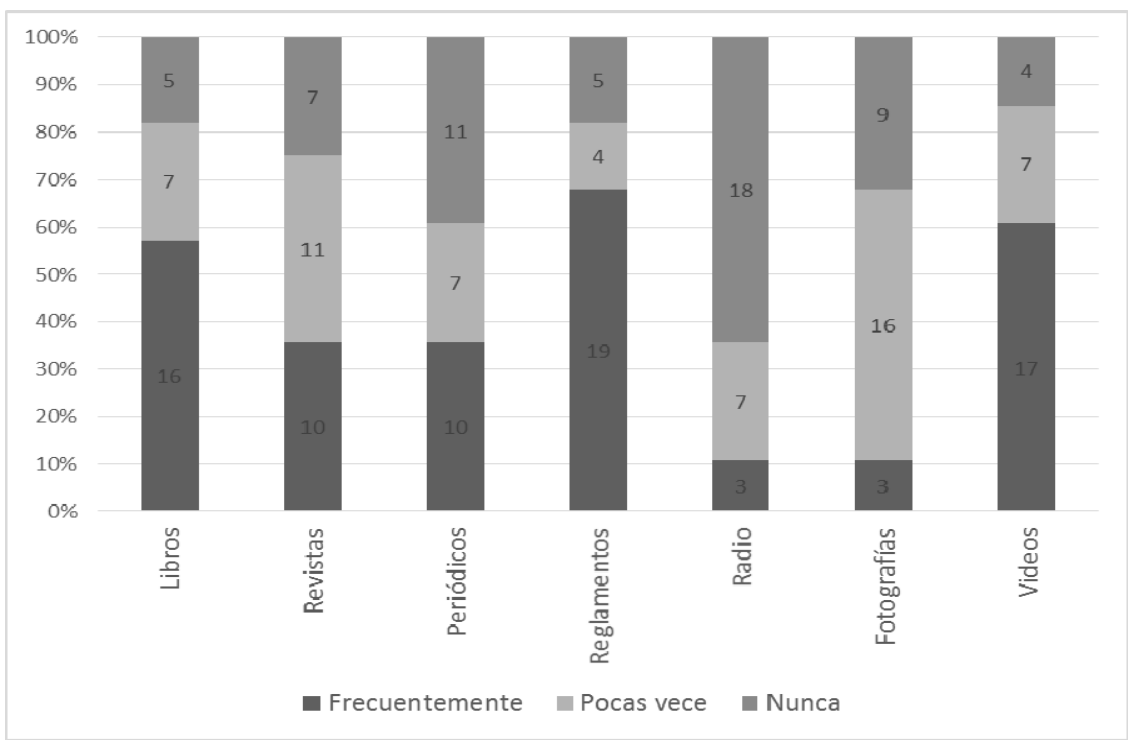

Los entrenadores en deportes de combate no se limitan a utilizar solo un recurso de información, como se delimito anteriormente, ya que usan desde su experiencia propia otros sujetos, el internet y libros. Además, cada recurso y fuente es consultado en diferente nivel, aunque siempre hay una inclinación en utilizar unos recursos más que otros para satisfacer su necesidad informativa.

\section{DISCUSIÓN DE RESULTADOS}

Como se ha visto hasta el momento, los resultados de este estudio muestran una mayor presencia del género masculino más que del femenino en los deportes. Lo que permitiría confirmar que los deportes de combate son dirigidos principalmente por hombres. Así mismo, sus representantes son sujetos de edad avanzada, es decir superan los cincuenta años, lo cual incide en su experiencia y tiempo de enseñanza, sin duda son características propias de esta comunidad.

Por otra parte, se tiene un alto índice de instructores con nivel académico de licenciados, probablemente se deba a que estos al encontrarse inmersos en instituciones educativas de nivel superior, haya sido requisito para laborar ahí como entrenadores deportivos. Cabe destacar, ninguno de los encuestados advirtió tener alguna licenciatura en el área de las ciencias del deporte. 
En cuanto a las necesidades de información de los entrenadores en deportes de combate encontramos que los principales temas de interés son: programas de entrenamiento, nutrición, motivación y técnicas de combate. Es indiscutible que el entrenador necesite información sobre programas de entrenamiento y técnicas de combate, gracias a la varianza de atletas que entrena, edad, sexo y objetivo de entrenamiento, sin duda, será distinta la dinámica cuando se enseña el deporte como actividad recreativa en comparación cuando los atletas participan en competencias. También la motivación juega un gran papel en el deporte, hacer que los atletas se comporten de determinada forma dentro y fuera del gimnasio, no es una tarea sencilla. La mayoría de los deportes de combate (boxeo, karate, taekwondo y lima lama) se clasifican por el peso de los individuos, de ahí que se tenga relevancia la nutrición. Es casi imposible instruir una disciplina deportiva si no se conocen programas de entrenamiento, técnicas de combate, motivación y nutrición.

Los resultados obtenidos concuerdan con lo advertido por Clarke (1995), menciona que la información sobre motivación de atletas y programas de entrenamiento son de alto interés por los instructores. En esta línea, Ermeling (2012) y Jones (2006) opinan que los conocimientos sobre temas de planificación y enseñanza de las técnicas deportivas son parte fundamental en la instrucción. Además, García (2006); Sagar y Jowett (2012) están de acuerdo en que el entrenador direcciona, orienta, motiva y controla la actividad de los atletas.

Cuando no se toman las precauciones mínimas para entrenar se corre el riesgo de tener una lesión y si el descuido es mayor puede llegar hasta la muerte. En los deportes de combate están más presente esta situación debido a que los encuentros pueden ser cuerpo a cuerpo o con un instrumento. Los entrenadores consideran importante el tema de las lesiones ya que están presentes continuamente, por lo cual se tenga que más de la mitad opine que el conocimiento de la prevención y rehabilitación de lesiones implica que debe poseer o buscar en un momento dado el entrenador. Nuestros resultados concuerdan con lo establecido por Hart, Trivedi, y Hutchinson, (2012) y Salerno (2009).

Un dato interesante es el obtenido en la forma en que los entrenadores $(n=26)$ se interesan más por el tópico de la nutrición y dietas de entrenamiento en comparación con lo relacionado a los suplementos alimenticios $(n=8)$ y bebidas rehidratantes $(n=6)$. Es posible que se haya llegado a este resultado a causa de que se necesita un conocimiento más especializado por parte de los instructores deportivos para diseminar la información que está en los envases de los suplementos y las bebidas deportivas. Hay que tener en cuenta que autores como: Christine, (2012); Pettersson et al, (2012); Pumpa et al., (2012) y Torres-McGehee, (2012) mencionan que se debe tener conocimiento de estos tres temas para lograr un óptimo desempeño del atleta.

En cuanto a las sustancias ilícitas y drogas es un tema interesante para los entrenadores y atletas durante competencias, debido a que en los torneos se ejecutan diversos exámenes médicos a los atletas representativos. En caso de encontrar resultados con alguna anomalía es seguro que el atleta sea descalificado. De ahí, radica la importan- 
cia de este tema pues si el entrenador desconoce el tópico de las drogas y sustancias ilícitas es difícil objetar o defender a sus atletas en caso de que sean inculpados por el uso de sustancias no permitidas. En esta investigación se pudo detectar que más de la mitad de los instructores deportivos $(n=15)$ indicaron requerir información sobre drogas y sustancias no permitidas, así mismo esto concuerda con lo expuesto con Hanstad y Loland (2009); Hanson (2009); Thomas et al, (2011) que advierten que los entrenadores llegan a desconocer información sobre drogas y sustancias ilícitas.

Sin embargo, casi un tercio de los entrenadores $(n=9)$ menciono requerir información sobre legislación deportiva, aunque esto no es tan destacado como lo había mencionado Wong (2010), en los deportes se consulta información de legislación deportiva. Si bien la mayoría de los entrenadores conoce así mismo usa los reglamentos pero es un hecho que tiene desconocimiento en la parte jurídica sobre asuntos concernientes al deporte.

Por otra parte, menos de un cuarto de los entrenadores $(n=7)$ encuestados señalo necesitar información sobre equipos y productos de competencia, records deportivos, sedes de competencia, rutas y medios de transporte. Aunque puede ser información que en un momento dado requiere el instructor deportivo, no es tan frecuente este tipo de necesidad informativa. Probablemente se deba a que en los torneos ya se tiene establecido el equipo y productos para competir, sedes de competencia, por lo tanto las rutas y medios de transporte llegan a estar predeterminadas. Aquí se destaca que los resultados obtenidos son similares a los de Vazquez y Calva (2013), donde encontraron que los boxeadores amateurs necesitan poca información sobre productos de competencia, sedes de competencia y records deportivos.

Con respecto al comportamiento informativo, la literatura señala que los entrenadores en general buscan y obtienen información por medio de la experiencia (Cushion, 2001; Cushion et al, 2003; Gilbert y Trudel, 2001 y Gould et al, 1990). Así que, el principal recurso de información utilizado por los entrenadores $(n=24)$ son ellos mismos y esto puede deberse a que tienen un aproximado entre 16 a 20 años enseñando alguna actividad deportiva además por el cumulo de conocimientos obtenidos a través de la experiencia, cursos e internet. Si bien es cierto que en esta investigación se encontró que la mayoría de los encuestados al tener estudios a nivel licenciatura ( $\mathrm{n}=17)$ y maestría $(\mathrm{n}=3)$ permiten se apoyen en el uso del internet y cursos, los cuales son medios con los que están familiarizados.

Desafortunadamente la comunidad de entrenadores en deportes de combate usan con poca frecuencia la biblioteca para consultar información. Sin embargo este hecho ha prevalecido además advertido por Clarke (1995) y más recientemente por Vazquez y Calva (2013) los cuales mencionan que la biblioteca no es el principal recurso para obtener información en las comunidades deportivas. En contraste nuestro análisis concuerda con Fowler (1976) y Callary et al, (2012) que indican que bastante información es adquirida por los cursos y seminarios.

En esta misma línea, se nota que los entrenadores tienen un comportamiento informativo similar a los investigadores en ciencias del deporte. Por su parte Guy 
(1979) confirma que los investigadores utilizan las fotografías y videos mientras que los entrenadores con frecuencia los frecuenta para obtener información, aunque no son sus principales fuentes para obtener información. Un dato curioso es el que se tiene del video que es el de mayor uso y concuerda con Wilson (2008) asimismo con Höferlin et al, (2010). Probablemente el deporte al aprenderse de forma práctica y al visualizar las técnicas de golpeo ayuda sin duda a mejorar los movimientos tanto de los entrenadores como del atleta.

Es cierto que nos encontramos que la biblioteca es poco explotada por los entrenadores, de modo que se pensaría que los libros no se usan, sin embargo nuestros resultados se obtuvo que más de la mitad de los instructores deportivos $(\mathrm{n}=15)$ frecuentemente consultan libros y en definitiva concuerda con lo previsto con Guy (1979) y Wright et al, (2007). Al parecer los libros que utilizan los instructores deportivos no los obtienen por medio de las bibliotecas, sino más bien corresponden a sus colecciones personales. Así mismo esto se confirma con el resultado obtenido, donde la mayoría de los entrenadores $(n=25)$ usan colecciones propias.

Se sabe que gran parte de la información se obtiene a través de medios impresos y digitales, no obstante las personas también pueden ser una fuente de información, de ahí que desde la perspectiva deportiva sean considerados los médicos, nutriólogos, psicólogos, masoterapeutas, fisioterapeutas entre otros especialistas como medios para buscar información (Lévesque,1991). En este estudio se pudo corroborar que los sujetos mencionados anteriormente proveen información, aunque entre los entrenadores en deportes de combate son de poco uso. Teniendo a consideración de que estos especialistas no se encuentran tan próximos en los gimnasios donde se localiza la mayoría de los instructores deportivos sino se ubican en institutos de medicina del deporte y consultorios médicos.

De forma general se encontró que muchos resultados obtenidos concuerdan con la literatura localizada en ciencias de la información, ciencias del deporte y medicina del deporte. Lo cual permite que el análisis de los entrenadores en deportes de combate sea una nueva rama en los estudios de usuarios así mismo comprobar que recursos están siendo utilizados por estos con el fin de que las unidades de información modifiquen la forma de proveer contenidos para la comunidad deportiva.

\section{CONCLUSIONES}

De acuerdo a lo encontrado sobre las necesidades de información y comportamiento informativo de los entrenadores en deportes de combate concluimos lo siguiente:

- La presencia de trabajos de carácter teórico así como práctico sobre necesidades de información y comportamiento informativo de comunidades deportivas vistas desde las ciencias de la información es bastante escasa, además enfocándose con entrenadores que se encuentran en proceso de formación. 
- A pesar de que la muestra es pequeña a causa de que se acudió directamente a los entrenadores que se dedican a enseñar algún deporte de combate y que no está en formación en un centro educativo para ser entrenador, se ofrece información sobre las necesidades y comportamiento informativo de los entrenadores empíricos.

- Con base en los datos obtenidos, se puede concluir que los instructores en deportes de combate manifiestan sus principales necesidades de información en los temas siguientes: programas de entrenamiento, nutrición y dietas de entrenamiento, motivación de atletas, así como técnicas de combate. En contraste, los temas que son de poco interés para los instructores es lo relacionado a los records deportivos, sedes de competencia, rutas y medios de transportes.

- Por lo que respecta a la forma en que el entrenador busca información casi todos los entrenadores se apoyan en su experiencia, internet y cursos. No obstante, los recursos que menos utilizan son: la información que proviene de familiares, atletas, jueces y árbitros, radio y periódicos.

- La biblioteca desafortunadamente no es el principal recurso para satisfacer la necesidad informativa entre los instructores deportivos. Así que, se confirma que los entrenadores no usan la biblioteca en primer instancia de modo que utilizan otros recursos como su experiencia, el internet y cursos para obtener información

- Se considera que los resultados obtenidos pueden ser de utilidad para aquellas unidades de información especializadas en deportes que desconocen al entrenador como usuario. De ahí que los indicadores encontrados en esta investigación se disponen para punto de partida para la toma de decisiones en el desarrollo de colecciones además en la generación, modificación y eliminación de algún servicio o producto logrando una reinvención en las bibliotecas deportivas.

\section{BIBLIOGRAFÍA}

CALLARY, B., WERTHNER, P. Y TRUDEL, P. (2012). How meaningful episodic experiences influence the process of becoming an experienced coach. Qualitative Research in Sport, Exercise and Health 4(3): 420-438. DOI: 10.1080/2159676X.2012.712985

CHRISTINE, K. (2012). Exploring the feasibility of an academic course that provides nutrition education to collegiate student-athletes. Journal of Nutrition Education and Behavior 44 (3): 267-270. DOI: 10.1016/j.jneb.2011.09.004

CLARKE, N. (1995). A study of information needs and information-seeking behavior of Australian accredited coaches in the sports of swimming and track and field, Master Dissertation. University of Canberra, Australian.

CUSHION, C. J., Armour, K. M. y Jones, R. L. (2003). Coach education and continuing professional development: experience and learning to coach. Quest 55(3): 215230. DOI: 10.1080/00336297.2003.10491800

CUSHION, C. J. (2001). Coaching research and coach education: Do the sum of the parts equal the whole? SportaPolis, September. 
IGLESIAS ANAYA, E. (2011). Planeación de una biblioteca deportiva: una propuesta para la comisión nacional de cultura física y deporte (CONADE). Tesis licenciatura, Escuela Nacional de Biblioteconomía y Archivonomía. Secretaría de Educación Pública.

ERMELING, B. A. (2012). Improving teaching through continuous learning: The inquirí process John Wooden used to become coach of the century. Quest 64: 197208. DOI: $10.1080 / 00336297.2012 .693754$

FOWLER, J. (1976). A survey of the profesional needs, interests and concerns of physical education teachers in Colorado. Association for health, physical education and recreation, Colorado University Departament of Physical Education.

GARCÍA UCHA, F. E. (2006). Psicología del deporte aplicada al rol del entrenador. Conferencia Magistral dictada en el I Congreso de la Sociedad Iberoamericana de Psicología del Deporte, ITESO Guadalajara.

GILBERT, W. Y TRUDEL, P. (2001). Learning to coach through experience: Reflection in model youth sport coaches. Journal of Teaching in Physical Education 21: 16-34.

GOULD, D., GIANINNI, J., KRANE, V. Y HODGE, K. (1990). Educational needs of elite U.S. national Pan American and Olympic coaches. Journal of Teaching in Physical Education (9): 322-344.

GUĐMUNDSSON, H. (2011). Swimming coaches' information seeking behaviour using the World Wide Web. Master Thesis, Faculty of Education Studies. University of Iceland.

GUY, R. F. (1979). The information needs and information seeking behavior of university lectures/researchs in the subjects areas of sport and physical education. Master Dissertation. University of Sheffield.

HANSON, J. M. (2009). Equipping athletes to make informed decisions about performance-enhancing drug use: a constructivist perspective from educational Psychology. Sport in Society: Cultures, Commerce, Media, Politics 12(3): 394-410. DOI:10.1080/17430430802673734

HANSTAD, D. V. Y LOLAND, S. (2009). Elite athletes' duty to provide information on their whereabouts: justificable anti-doping work or an indefensible surveillance regime? European Journal of Sport Science 9(1): 3-10. DOI:10.1080/17461390802594219

HART, M. G., TRIVEDI, R. A. Y HUTCHINSON, P. J. (2012). Boxing sparring complicated by an acute subdural haematoma and brainstem haemorrhage. British Journal of Neurosurgery 26 (5): 776-778. DOI:10.3109/02688697.2012.657270

HERNÁNDEZ SALAZAR, P. (2001). La producción del conocimiento científico como base para determinar perfiles de usuarios. Investigación Bibliotecológica, 15 (30):29-64.

HÖFERLIN, M., GRUNDY, E., BORGO, R., WEISKOPF, D., CHEN, M., GRIFFITHS, I. W. Y GRIFFITHS, W. (2010). Video Visualization for Snooker 
Skill Training. Eurographics/ IEEE-VGTC Symposium on Visualization 29 (3): 1053- 1062. DOI: 10.1111/j.1467-8659.2009.01670.x

KRIKELAS, J. (1983). Information-seeking behavior: patterns and concepts. Drexel Library Quaterly 19:5-20.

KUHLTHAU, C.C. (1991). Inside the search process: information seeking from the user's perspective. Journal of the American Society for Information Science 42(5):361-371.

DOI: 10.1002/(SICI)1097-4571(199106)42:5<361::AIDASI6>3.0.CO;2-\#

JONES, R. L. (2006). The sports coach as educator: Re-conceptualising sport coaching. United Kingdom: Routledge, Taylor and Francis.

LÉVESQUE, D. (1991). El entrenamiento en los deportes. España: Pixel.

MARTENS, R., CHRISTINA, R. W., HARVEY, J. S. Y SHARKEY, B. J. (1995). El entrenador: Nociones sobre psicología, pedagogía, fisiología y medicina para conocer el éxito. España: Hispano Europea.

PETTERSSON, S., PIPPING EKSTRÖM, M. Y BERG, C. M. (2012). The food and weight combat. A problematic fight for the elite combat sports athlete. Appetite 59: 234-242. DOI:10.1016/j.appet.2012.05.007

PUMPA, K. L., MADIGAN, S. M., WOOD-MARTIN, R.E., FLANAGAN, R. Y ROCHE, N. (2012). The development of nutritional-supplement fact sheets for Irish athletes: A case study. International Journal of Sport Nutrition and Exercise Metabolism 22: 220 -224.

REMANS, A. (2000). Información y documentación deportiva en Europa. Revista General de Información y Documentación Deportiva 10 (1): 17-29.

SAGAR, S. S. Y JOWETT, S. (2012). Communicative acts in coach-athlete interactions: When losing competitions and when making mistakes in training. Western Journal of Communication 76(2): 148-174. DOI: 10.1080/10570314.2011.651256

SALERNO, R. R. (2009). Sport injuries and their effects on health. New York: Nova Science Publishers.

SANZ CASADO, E. (1994). Manual de estudios de usuarios. Madrid:Fundación Germán Sánchez Ruipérez-Pirámide.

THOMAS, J. O., DUNN, M., SWIFT, W. Y BURNS, L. (2011). Illicit drug knowledge and information-seeking behaviours among elite athletes. Journal of Science and Medicine in Sport 14: 278-282. DOI: 10.1016/j.jsams.2011.02.001

TORRES-MCGEHEE, T. M., PRITCHETT, K. L., ZIPPEL, D., MINTON, D. M., CELLAMARE, A. Y SIBILIA, M. (2012). Sports nutrition knowledge among collegiate athletes, coaches, athletic trainers, and strength and conditioning specialists. Journal of Athletic Training 47(2):205-211.

VARGAS, Rene. 1998. Teoría de entrenamiento: diccionario de conceptos. México: UNAM.

VAZQUEZ-MOCTEZUMA, S. E. Y CALVA GONZÁLEZ, J. J. (2013). Análisis de las necesidades de información y comportamiento en la búsqueda de información 
de atletas amateurs: los boxeadores. Investigación Bibliotecológica 27 (61): 175206

WILSON, B. D. (2008). Development in video technology for coaching. Sport Technol 1(1): 34-40. DOI: $10.1002 /$ jst.9

WILSON, T. D. (2000). Human information behavior. Information Science 3 (2): 4955.

WONG, G. M. (2010). Essentials of sports law. $4^{\circ}$ ed., California: Praeger.

WRIGHT, T., Trudel, P. y Culver, D. (2007). Learning how to coach: the different learning situations reported by youth ice hockey coaches. Physical education and sport pedagogy 12 (2): 127-144. DO 